\title{
EQUATIONS IN FREE METABELIAN GROUPS
}

\author{
ROGER C. LYNDON ${ }^{1}$
}

Baumslag and Mahler [1] have shown that, for $F$ a free group and hence $G=F / F^{\prime \prime}$ a free metabelian group, and $p$ any prime, the relation $a^{p} b^{p}=c^{p}$ cannot hold for elements $a, b$, and $c$ of $G$ such that $a G^{\prime}$ and $b G^{\prime}$ are independent elements of the free abelian group $G / G^{\prime}$. In answer to a question they raised, we show by their methods that, if $p, q$, and $r$ are three primes, not all the same, then there exist solutions of the equation $a^{p} b^{q}=c^{r}$ in $G$, with $a$ and $b$ independent modulo $G^{\prime}$.

We may suppose that $r \neq p, q$. If such a solution exists at all, one exists in $G$, free metabelian on two generators $x$ and $y$, and such that, modulo $G^{\prime}, a \equiv x^{m r}, b \equiv y^{n r}$, and $c \equiv x^{m p} y^{n q}$, for some positive integers $m$ and $n$. Let $L$ be the ring of Laurent polynomials over the integers in $x$ and $y$ (that is, admitting both positive and negative integer exponents). Then $G^{\prime}$ is naturally the free $L$ module with generator $k=x^{-1} y^{-1} x y$, that is, with $u^{x}=x^{-1} u x, u^{y}=y^{-1} u y$, and $u^{A+B}=u^{A} u^{B}$, for all $u$ in $G^{\prime}$ and $A, B$ in $L$. In this notation, we have

$$
a=x^{m r} k^{A}, \quad b=y^{n r} k^{B}, \text { and } c=x^{m p} y^{n q} k^{C},
$$

for certain elements $A, B$, and $C$ of $L$. Let $\Gamma_{h}(z)$ be the cyclotomic polynomial with roots all primitive $h$ th roots of unity. The condition $a^{p} b^{q}=c^{r}$ reduces by straightforward computation to the condition on $A, B$, and $C$ that

$$
A \Gamma_{p}\left(x^{m r}\right) y^{n r}+B \Gamma_{q}\left(y^{n r}\right)=C \Gamma_{r}\left(x^{m p} y^{n q}\right)+D(x, v),
$$

where

$D(x, y)=\left(1+x+\cdots+x^{m p-1}\right)\left(1+y+\cdots+y^{n q-1}\right) x^{-m p} E\left(x^{m p}, y^{n q}\right)$, and

$$
E(u, v)=\sum_{i=0}^{r-1}(u v)^{i} \frac{u^{r-i}-1}{u-1} .
$$

Collecting terms in $X=x^{m}$ and $Y=y^{n}$ gives an equation

$$
A_{1} \Gamma_{p}\left(X^{r}\right) Y^{r}+B_{1} \Gamma_{q}\left(Y^{r}\right)=C_{1} \Gamma_{r}\left(X^{p} Y^{q}\right)+X^{-p} D_{1}(X, Y)
$$

where

Received by the editors October 4, 1965.

${ }^{1}$ Work supported in part by a grant from the National Science Foundation. 


$$
D_{1}(X, Y)=\Gamma_{p}(X) \Gamma_{q}(Y) E\left(X^{p}, Y^{q}\right) .
$$

It is easy to see that, conversely, the existence of a solution $A_{1}, B_{1}, C_{1}$ of this last equation implies that of a solution of $a^{p} b^{q}=c^{r}$ with $a$ and $b$ independent modulo $G^{\prime}$.

It remains, then, to show that $D_{1}$ belongs to the ideal in $L$ generated by $\Gamma_{p}\left(X^{r}\right), \Gamma_{q}\left(Y^{r}\right)$, and $\Gamma_{r}\left(X^{p} Y^{q}\right)$. Since all elements concerned lie in the polynomial ring $Z[X, Y]$, this comes to showing that $D_{1}$ belongs to the ideal $J$ of this ring generated by the same three elements. Since $\Gamma_{p}\left(X^{r}\right)=\Gamma_{p r}(X) \Gamma_{p}(X)$, the ring $Z[X] / \Gamma_{p}\left(X^{r}\right)$ decomposes into $Z[X] / \Gamma_{p r}(X)$ and $Z[X] / \Gamma_{p}(X)$, and $Z[X, Y] / J$ decomposes correspondingly. Since $\Gamma_{p}(X)$ divides $D_{1}(X, Y)$, it suffices to consider the first component only. A similar argument for $Y$ enables us to replace $J$ by the ideal $J_{1}$ generated by $\Gamma_{p r}(X), \Gamma_{q r}(Y)$, and $\Gamma_{r}\left(X^{p} Y^{q}\right)$.

We may identify $Z[X] / \Gamma_{p r}(X)$ with the ring $Z[\xi]$ where $\xi$ is a primitive $p r$ th root of unity. Over this ring, $\Gamma_{q r}(Y)$ splits into factors $\left(Y^{q}-\omega\right) /(Y-\omega)$, where $\omega$ runs through the $r-1$ primitive $r$ th roots of unity. Therefore $Z[X, Y]$ is a subdirect product of rings $Z[\xi, \eta]$ of algebraic integers, where $\xi$ runs through the primitive prth roots of unity and $\eta$ through the primitive $q r$ th roots of unity. We shall show that, in each such ring, $\gamma=\Gamma_{r}\left(\xi^{p} \eta^{q}\right)$ divides $\delta=\Gamma_{p}(\xi) \Gamma_{q}(\eta) E\left(\xi^{p}, \eta^{q}\right)^{\cdot}$

Both $\omega=\xi^{p}$ and $\zeta=\eta^{q}$ are primitive $r$ th roots, and we consider two cases, according as $\omega \zeta=1$ or not. If $\omega \zeta \neq 1$, then $\omega \zeta$ is a primitive $r$ th root, whence we find that

$$
E(\omega, \zeta)=\sum_{0}^{r-1} \frac{\omega^{r} \zeta^{i}-(\omega \zeta)^{i}}{\omega-1}=\left(\sum \zeta^{i}-\sum(\omega \zeta)^{i}\right) /(\omega-1)=0,
$$

that $\delta=0$, and so $\gamma$ divides $\delta$. Suppose henceforth that $\omega \zeta=1$. Then $\gamma=\Gamma_{r}(1)=r$, while

$$
E(\omega, \zeta)=\sum \frac{\omega^{-i}-1}{\omega-1}=\left(\sum \omega^{-i}-\sum 1\right) /(\omega-1)=-r /(\omega-1),
$$

and hence

$$
\delta=\Gamma_{p}(\xi) \Gamma_{q}(\eta) E(\omega, \zeta)=\frac{\omega-1}{\xi-1} \frac{\omega-1}{\eta-1} \frac{-r}{\omega-1}=-r \frac{\omega-1}{(\xi-1)(\eta-1)} .
$$

It suffices to show that $\xi-1$, and similarly $\eta-1$, are units. Since $\xi$ has minimal polynomial

$$
\Gamma_{p r}(z)=\frac{z^{p r}-1}{z^{p}-1} / \frac{z^{r}-1}{z-1}
$$


the number $\xi-1$ has minimal polynomial $P(z)=\Gamma_{p r}(z+1)$, and hence norm $P(0)=\Gamma_{p r}(1)=1$, and is therefore a unit. This provides $U(X, Y)$ in $Z[X, Y]$ such that $D_{1}(\xi, \eta)=U(\xi, \eta) \Gamma_{r}\left(\xi^{p} \eta^{p}\right)$ holds for $\xi$ and $\eta$ as in the case last considered; it is easily checked that the same hold if $\xi$ and $\eta$ are any roots of $\Gamma_{p}\left(X^{r}\right)$ and $\Gamma_{q}\left(Y^{r}\right)$, whence it follows that this equation holds in $Z[X, Y] /\left(\Gamma_{p}\left(X^{r}\right), \Gamma_{q}\left(Y^{r}\right)\right)$, and that $D_{1}$ is in $J$. This completes the proof.

\section{BIBLIOGRAPHY}

1. Gilbert Baumslag and Kurt Mahler, Equations in free metabelian groups, Michigan Math J. 12 (1965), 417-420.

Queen Mary College, London and

University of Michigan 